\title{
Sliding Mode Controller for Three-Phase Hybrid Active Power Filter with Photovoltaic Application
}

\author{
Ayman Blorfan $^{1 *}$, Jean Mercklé ${ }^{1}$, Damien Flieller ${ }^{2}$, Patrice Wira ${ }^{1}$, Guy Sturtzer ${ }^{2}$ \\ ${ }^{1}$ Université de Haute Alsace, MIPS Laboratory, Mulhouse, France; ${ }^{2}$ INSA de Strasbourg, GREEN Laboratory, Strasbourg, France. \\ Email: *ayman.blorfan@uha.fr
}

Received November $30^{\text {th }}, 2011$; revised January $30^{\text {th }}, 2012$; accepted February $8^{\text {th }}, 2012$

\begin{abstract}
This paper presents a new three-phase hybrid active power filter configuration that interconnects a passive high-pass filter in parallel with an active power filter and a photovoltaic system. The proposed configuration can improves the filtering performance of the conventional active power filter, as well as simultaneously supply the power from the photovoltaic arrays to the load and utility. This paper will describe the proposed hybrid active power filter control using sliding mode with photovoltaic system. The proposed technique effectively filters harmonics under $1 \mathrm{kHz}$ but also higher frequency to achieve wideband harmonics compensation. The THD of source current is reduced from $30.09 \%$ to $1.95 \%$. The result indicates that the sliding mode controller can track the reference signals and have good dynamic characteristics.
\end{abstract}

Keywords: Hybrid Active Power Filter; p-q Theorem; Photovoltaic; Boost Converter; Wideband Harmonic Compensation

\section{Introduction}

The past several decades have seen a rapid increase of power electronics-based loads connected to the utility system [1]. However, the proliferation of these non-linear loads has raised concern with regard to the resulting harmonic distortion levels of the supply current on the power system [2]. The general scheme of the power active filter is shown in Figure 1. Passive filtering is the traditional method of harmonic filtering. It is well known that the application of passive filters creates new system resonances that are dependent on the specific system conditions. Although this solution is simple, it has brings

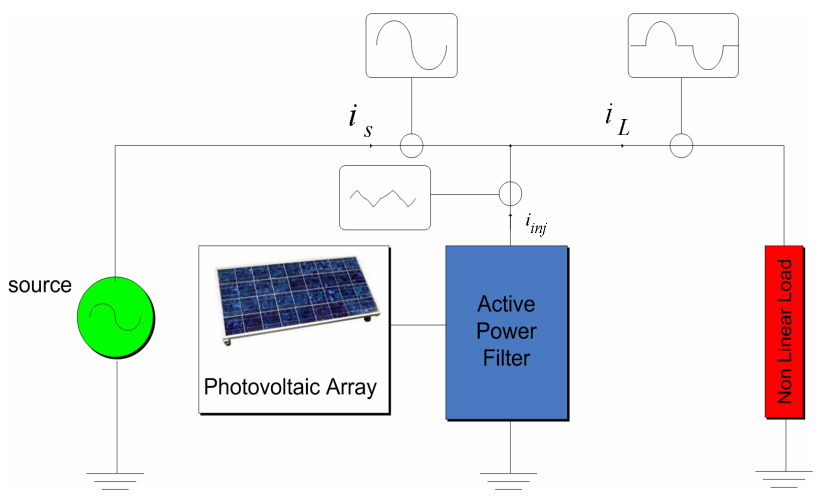

Figure 1. The general Scheme of the PAF.

"Corresponding author. rise to several shortcomings. Furthermore, since the harmonics that to be eliminated are of low order, large filter components are required [3].

The sliding mode control has been widely applied to power converters due to its operation characteristics such as fastness, robustness and stability for large load variations.

Hybrid APFs were developed, where a passive filter is connected parallel to a conventional APF [4]. The hybrid APF configuration is effective in improving the damping performance of high-order harmonics. Our system consists of five parts:

1) The source: $A C$ line sinusoidal balanced or not.

2) A Photovoltaic Array: A 9-module ( $85 \mathrm{~W}$ each) PV array $\left\{\right.$ sun $\left(1000 \mathrm{~W} / \mathrm{m}^{2}\right.$ insolation $)+$ A Boost DC-DC converter\}.

3) A Non-linear load: bridge diode rectifier feeding an $\mathrm{RL}$ inductive load.

4) A three phase inverter.

5) An RLC passive power filter.

Recently, there is an increasing concern about the environment pollution. The need to generate pollution-free energy has trigger intensive considerable effort toward alternative source of energy. Solar energy, in particular, is a promising option. Some researcher had spent their effort in developing the combined system of an APF and a photovoltaic (PV) system [5,6]. However, the existing 
hybrid APF configurations with there benefits are not yet implemented for the PV application.

\section{Principle of the Sliding Mode Control}

The sliding mode design approach consists of two components. The first involves the design of a switching function so that the sliding motion satisfies the design specifications. The second is concerned with the selection of a control law, which will make the switching function attractive to the system state. It can be seen that this control law is not necessarily discontinuous. Considering the following general system with scalar control [7]. Let the nonlinear system of the form

$$
\dot{X}=f(x, u)
$$

The second member of the equation is discontinuous due to the discontinuity imposed on the control side of the surface $\sigma(x)=0$. Where $X$ is the system error and $\mathrm{u}$ is the control issued by the corrector with variable structure. Depending on the order applied, the system will take one of two forms below and shown in Figure 2.

$$
\begin{gathered}
X=f^{--}\left(X, U^{--}\right) \text {if } \sigma(X)<0 \\
X=f^{+}\left(X, U^{+}\right) \text {if } \sigma(X)>0
\end{gathered}
$$

A nonlinear system is given by the differential equation below:

$$
\dot{X}=f(X, t)+g(X, t) \cdot U
$$

where,

$$
X \in \mathfrak{R}^{n}, U \in \mathfrak{R}^{m}, f(X, t) \in \mathfrak{R}^{n}, g(X, t) \in \mathfrak{R}^{n \times m}
$$

How to apply the variable structure for the command? Two questions can be asked:

1) What is this control?

2) How does it synthesize?

The synthesis of a variable structure represented by Figure 3 involves two phases [8]:

- The first is to define the switching surface $\sigma(x)=0$ of

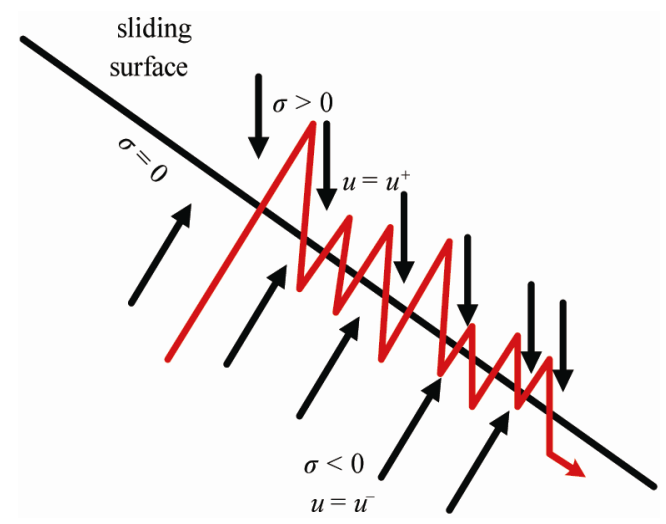

Figure 2. System behaviors on the sliding surface. desired dynamics.

- The second aims to synthesize the control function $u(x, t)$, in order to make sure that any state outside the switching surface will be joined to the states inside the sliding tranche (with bandwidth $2 \delta$ surrounding the sliding surface, see the Figure 4) during a finite time (limited by the switching frequency of the IGBT). Once it reaches, a sliding mode occurs. The system adopts the dynamic of the surface and the point representing the evolution of the system reaches the equilibrium point.

The dynamic slip is independent of the command that serves only the evolution of the intersection of surfaces and ensure their attractiveness. The control synthesis is realized when:

The origin of coordinates in space of the system is a stable equilibrium point. From any initial condition, the representative point of the movement meets the hypersurface slip in a finite time.

\section{Choice of the Sliding Surface}

Generally, the sliding surfaces are chosen in as being hyper plants through the origin of space and general form

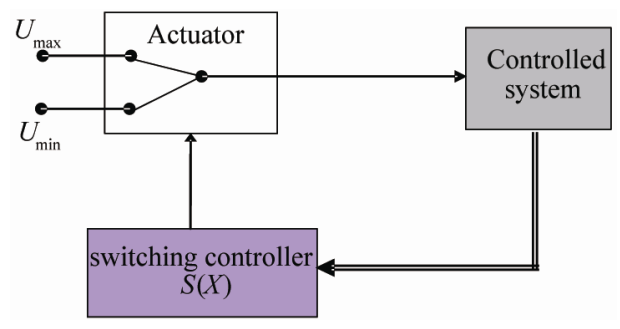

Figure 3. Schematic diagram of variable structure control.
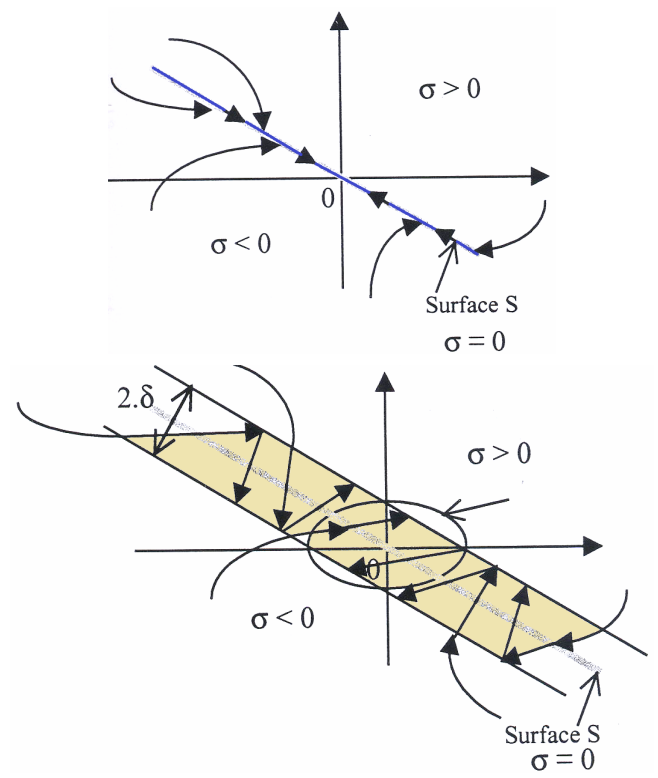

Figure 4. Trajectories of sliding mode, ideal and real. 
order less than the given system and representing the given by:

$$
\begin{aligned}
& \sigma(x)=C x=0 \\
& \sigma(x)=\sum_{i=1}^{n} c_{i} x_{i}
\end{aligned}
$$

Although the surface may be nonlinear, the linear surfaces are more practical for the design of corrective variable structure sliding mode in case the system is multivariable.

Given

$$
\dot{X}=f(X, t)+g(X, t) \cdot U
$$

where,

$$
X \in \mathfrak{R}^{n}, U \in \mathfrak{R}^{m}, f(X, t) \in \mathfrak{R}^{n}, g(X, t) \in \mathfrak{R}^{n \times m}
$$

The problem is more complex due to existence of several switching surfaces $\left(\sigma_{1} \sigma_{2} \sigma_{3} \cdots \sigma_{m}\right)$. The sliding mode occurs on the intersection of these surfaces.

The two switching surfaces are chosen as:

$$
\begin{gathered}
\sigma(x)=C x \\
\sigma(x)=\left[\sigma_{1}(x), \sigma_{2}(x), \sigma_{m}(x)\right]^{T}=0 \\
C=\left[c_{1}, c_{2}, \cdots c_{n}\right]
\end{gathered}
$$

The switching surface $\sigma(x)=0$ is a surface of dimension " $m$ " and $\sigma_{i}(x)$ is the ith switching surface. The intersection of the surface $m(x)$ is a sliding surface dimensions $(n-m)$. Each entry has the form $U_{i}$ :

$$
\begin{gathered}
U(x, t)=\left\{\begin{array}{l}
U^{+} \text {avec } \sigma_{i}(x)>0 \\
U^{-} \text {avec } \sigma_{i}(x)<0
\end{array}\right. \\
i=1, \cdots, m
\end{gathered}
$$

The switching surface is chosen such that when the system is in sliding mode, the behavior is meeting the desired objectives. After selecting the sliding surface, an important phase remains to consider. Can we ensure the existence of a sliding mode?

If the tangents to the trajectories or velocity vectors near the surface are directed towards the switching surface $\sigma(x)=0$, then a sliding mode occurs on this plane.

Given the canonical form:

$$
\left\{\begin{array}{l}
\dot{x}_{i}=x_{i+1} \quad i=1, \cdots, n-1 \\
\dot{x}_{n}=-\sum_{i=1}^{n} a_{i} x_{i}+f(t)+u
\end{array}\right.
$$

where $u$ is the command with discontinuities in the vicinity of $\sigma(x)=0$.

(The disturbance $f(t)$ and parameters may be unknown.)

The switching surface is of the form:

$$
\sigma(x)=\sum_{i=1}^{n} c_{i} x_{i}
$$

where the coefficients are constants. The pair of following inequalities constitutes the conditions to the existence of the sliding-mode:

$$
s \underline{\lim }_{0^{-}} \dot{\sigma}>0 \text { et } \quad \underline{\lim }_{0^{+}} \dot{\sigma}<0
$$

To prove the invariability with respect to the coefficient $\mathbf{a}_{\mathbf{i}}$ and $\mathbf{f}$, solve the equation $=0$, and replace it in (12).

Once the system is in sliding mode, the closed loop system becomes:

$$
\left\{\begin{array}{l}
\dot{x}_{i}=x_{i+1} \quad i=1, \cdots, n-2 \\
\dot{x}_{n-1}=-\sum_{i=1}^{n-1} c_{i} x_{i}
\end{array}\right.
$$

The system no longer dependent neither on $f(t)$ and nor on the $a_{i}$ coefficients, contrariwise it depends on the $c_{i}$, which gives the system an invariance to disturbances and parametric variations.

Let the system described by Equation (15), and a surface $\sigma$ separating the hyper-surface into two parts $\mathrm{G}^{+}$ $\mathrm{G}^{-}$.

$$
\frac{\mathrm{d} X}{\mathrm{~d} t}=F\left(t, x_{1}, \cdots, x_{n}\right)
$$

with $X=\left[x_{1}, \cdots, x_{n}\right]^{T} ; F=\left[f_{1}, \cdots, f_{n}\right]^{T}$. The functions $f_{1}, \cdots, f$ are piecewise continuous and have discontinueties on the hyper surface $\sigma$ of the equation $\sigma(x)=0$.

$f_{N}^{+}, f_{N}^{-}$the $f$-projections on the normal oriented of $\sigma$. These projections are made on both sides of the surface, we can show that if at any point on the surface $\sigma$, $\frac{\partial f_{i}}{\partial x_{j}}$ is bounded, $f_{N}^{+}-f_{N}^{-} \quad\left(f_{N}^{+}<0\right.$ et $\left.f_{N}^{-}>0\right)$ is negative, and the following inequality $\sigma \dot{\sigma}<0$ holds.

The sliding condition can take two equivalent forms given by equations below:

- $\sigma \dot{\sigma}<0$

- $f_{N}^{+}<0$ being $\sigma>0$ and $\sigma \dot{\sigma}<0$

- $f_{N}^{-}>0$ being $\sigma<0$ and $\dot{\sigma}>0$

where $\left(f_{N}^{+}<0\right.$ et $\left.f_{N}^{-}>0\right) \Rightarrow \sigma \dot{\sigma}<0$

In the ideal sliding mode, the switching frequency is assumed to be infinite. In practice the frequency is limited by the components of power electronics. The introduction of hysteresis does not call into question the theory of sliding but this hysteresis increases the phenomenon of "chattering" embodied by the oscillations around the sliding surface. Figure 4 shows the effect of hysteresis bandwidth $2 \delta$. The balance point is arbitrarily chosen as origin. Commutations move freely within the bandwidth around the surface. A Phenomenon appears around the equilibrium point. These trajectories are shown in Figure 4. 
Consider the system mono-variable described by the equation of state below:

$$
\dot{X}=f(X, t)+g(X, t) \cdot U
$$

where,

$$
X \in \mathfrak{R}^{n}, U \in \mathfrak{R}^{m}, f(X, t) \in \mathfrak{R}^{n}, g(X, t) \in \mathfrak{R}^{n \times m}
$$

The equivalent control of the ideal sliding mode (neither threshold, neither delay, neither hysteresis) is obtained on $\sigma(x, t)=0$ with $\dot{\sigma}=(x, t)=0$.

$$
\begin{aligned}
\dot{\sigma} & =(x, t)=\left(\frac{\partial \sigma}{\partial x}\right)^{T} \frac{\mathrm{d} x}{\mathrm{~d} t}+\frac{\partial \sigma}{\mathrm{d} t} \\
& =\left(\frac{\partial \sigma}{\partial x}\right)^{T}\left[f(x, t)+g(x, t) U_{e q}\right]+\frac{\partial \sigma}{\mathrm{d} t} \\
U_{e q}(x, t) & =-\left[\left(\frac{\partial \sigma}{\partial x}\right)^{T} g(x, t)\right]^{-1}\left\{\left(\frac{\partial \sigma}{\partial x}\right)^{T} f(x, t)+\frac{\partial \sigma}{\partial t}\right\}
\end{aligned}
$$

The condition of sliding mode:

$$
\left[\left(\frac{\partial \sigma}{\partial x}\right)^{T} g(x, t)\right]^{-1} \neq 0
$$

This condition is also called the transversality condition. In reality the fact that the product is zero means that the vector field $g$ is orthogonal to the surface normal vector. So $g$ has no vertical component and system status can not be brought to the surface. This condition is necessary but not sufficient to ensure the sliding mode.

By replacing the expression of the equivalent control in the system of Equations (19), we obtain the following equation:

$$
\begin{aligned}
\dot{x}= & \left\{I-g(x, t)\left[\left(\frac{\partial \sigma}{\partial x}\right)^{T} g(x, t)\right]^{-1}\left(\frac{\partial \sigma}{\partial x}\right)^{T}\right\} f(x, t) \\
& -g(x, t)\left[\left(\frac{\partial \sigma}{\partial x}\right)^{T} g(x, t)\right]^{-1}\left(\frac{\partial \sigma}{\partial x}\right)^{T}
\end{aligned}
$$

The equivalent control shown in Figure 5 maintains the system state on the sliding surface $\sigma=0$. The average value that takes control is defined as follows:

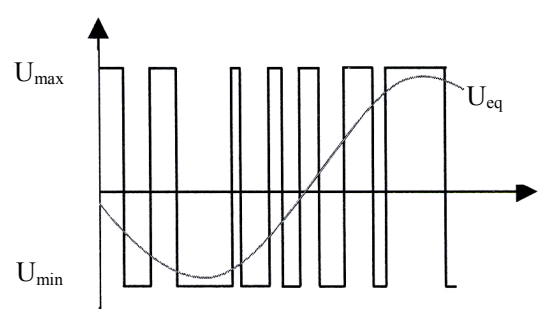

Figure 5. Representation of the equivalent control.

$$
\min \left\{U^{-}(x, t), U^{+}(x, t)\right\}<u_{e q}<\max \left\{U^{-}(x, t), U^{+}(x, t)\right\}
$$

The necessary and sufficient condition for existence of sliding mode is given by:

$$
\begin{aligned}
& U_{\text {min }}=\min \left\{U^{-}(x, t), U^{+}(x, t)\right\} \\
& U_{\text {max }}=\max \left\{U^{-}(x, t), U^{+}(x, t)\right\}
\end{aligned}
$$

\section{Determination of the Reference Current}

The dynamic performance of the system will also depend on the different algorithms used for controlling the active filter [9]. The method adopted to extract the reference currents and the synthesis of different regulators (control loops of voltage and current) will ensure a sinusoidal current on the network. Different methods of identifying harmonic (or fundamental) current components of nonlinear load can be used. In what follows, we will present and analyze respectively the $p-q$ method of instantaneous power and the direct method. Their use will depend on the targets: harmonic compensation, harmonic compensation, its application to three-phase installations (balanced or unbalanced) or single phase. This technique is illustrated by Figure 6.

In order to generate the compensation current that follows the current reference, the fixed-band hysteresis current control method is adopted. The proposed scheme is controlled to inject the reactive and harmonic current of the nonlinear load and the reactive current of the passive filter. Furthermore, a current must be drawn from the utility source to maintain the voltage across the DC-bus capacitor to a preset value that is higher than the amplitude of the source voltage. Under the normal operation, the PV system will provide active power to the load and the utility.

Under poor PV power generation condition, the utility source supplies the active power to the load directly.

\section{Characteristic of the System}

The power circuit of the proposed hybrid APF with PV system, in parallel with a nonlinear load, that is supplied by source voltage from the point of common coupling

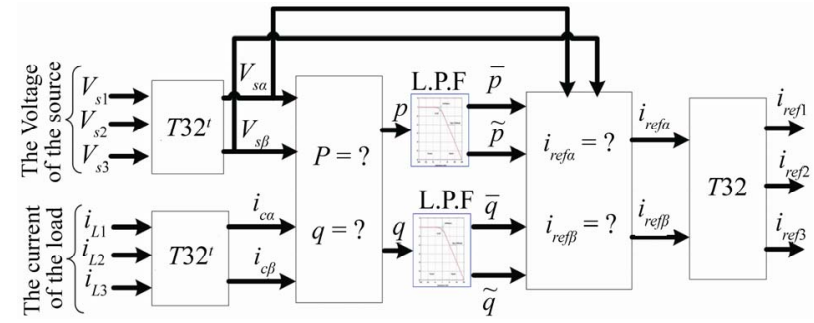

Figure 6. Overall system configuration and control block diagram. 
PCC. The proposed hybrid APF consists of a passive high-pass filter (HPF), a shunt APF constructed by a three-phase full-bridge voltage source inverter (VSI) connected to a DC-bus capacitor; and PV arrays in parallel with the DC-bus capacitor. In the proposed scheme, the low order harmonics are compensated using the shunt APF, while the high-order harmonics are filtered with the passive HPF. This configuration is effective to improve the filtering performance of the high-order harmonics. The inverter is shown in Figure 7.

The simplest solar cell model consists of diode and current source parallel connected. Source current is directly proportional to the solar radiation. Diode represents PN junction of a solar cell.

$$
\begin{gathered}
i_{P V}=I_{S C}-i_{D} \\
i=I_{0}\left(e^{\frac{e V}{K T}}-1\right)-I_{L} \Rightarrow i=I_{0}\left(e^{\frac{V_{D}}{V_{T}}}-1\right)-I_{D}
\end{gathered}
$$

The proposed model of a photovoltaic cell using Matlab is shown by Figure 8.

The photovoltaic system consists of:

1) A 9-module (85 W each) PV array with full sun $\left(1000 \mathrm{~W} / \mathrm{m}^{2}\right.$ insolation);

2) A Boost DC-DC converter that steps-up a DC input voltage;

3) A capacitor C under DC voltage that provides necessary energy storage to balance instantaneous power delivered to the grid.

The photovoltaic system is shown by Figure 9 .

The pollution load is materialized by a bridge diode rectifier feeding an RL inductive load. The network is modeled by three perfect sinusoidal voltage sources in series with an inductance Ls and resistance Rs Bridge operates under a simple rms voltage of $220 \mathrm{~V}$ and consumes a power rating of 5.2 KVA. We deduce the following characteristic quantities for the nonlinear load:

$$
V_{d}=\frac{3 \times V_{s} \times \sqrt{6}}{\pi}=514.86 \mathrm{~V},
$$

$$
I_{d}=\frac{S_{n}}{V_{d}}=10 \mathrm{~A}, R_{d}=\frac{V_{d}}{I_{d}}=52 \Omega
$$

The inductor $L$ has a value between $1 \%$ and $10 \%$ of the nominal value of inductance $L$ is defined by:

$$
L=\frac{V_{s}}{\omega \times I_{c 1}} \text { with } I_{c 1}=\sqrt{\frac{2}{3}} I_{d}
$$

The term $I_{c 1}$ represents the effective value of the fundamental component of the load current $I_{c}$ and $\omega$ the pulse of the electrical network.

\section{Control the Switching Frequency}

The parametric study has confirmed that the switching frequency is strongly dependent on coupling inductance Lf and width of hysteresis band. The objective of this part is to propose a method to limit the frequency switching and make it independent of parametric variations. For this, we propose to add to the expression of the surface a triangular signal whose frequency is constant and the amplitude is fixed. The principle of the method is illustrated in Figure 10.

\section{Simulation Results}

The proposed hybrid APF was simulated using MAT-

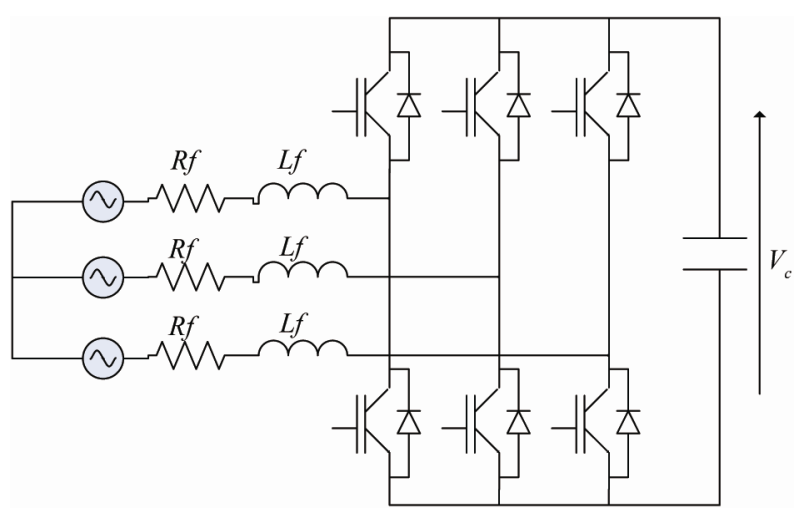

Figure 7. Structure of the inverter.

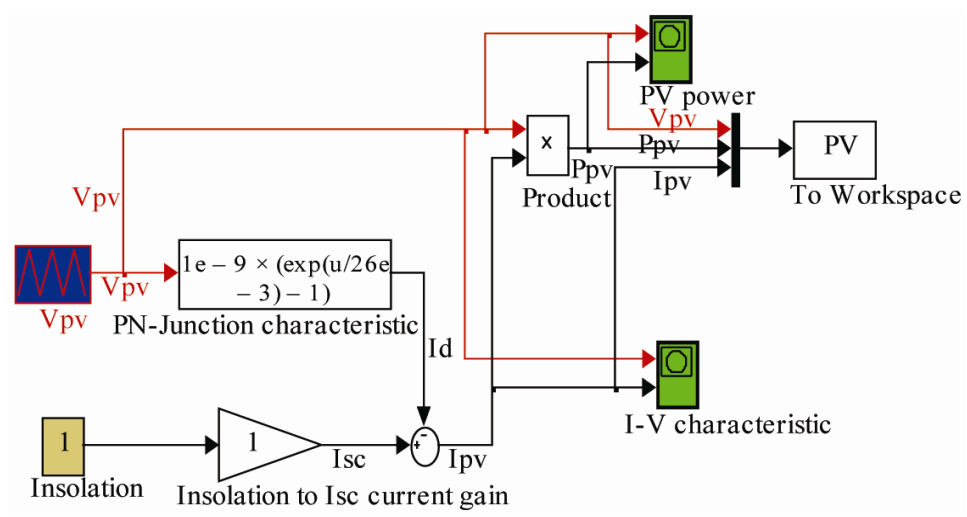

Figure 8. Model of PV cell using simulink. 


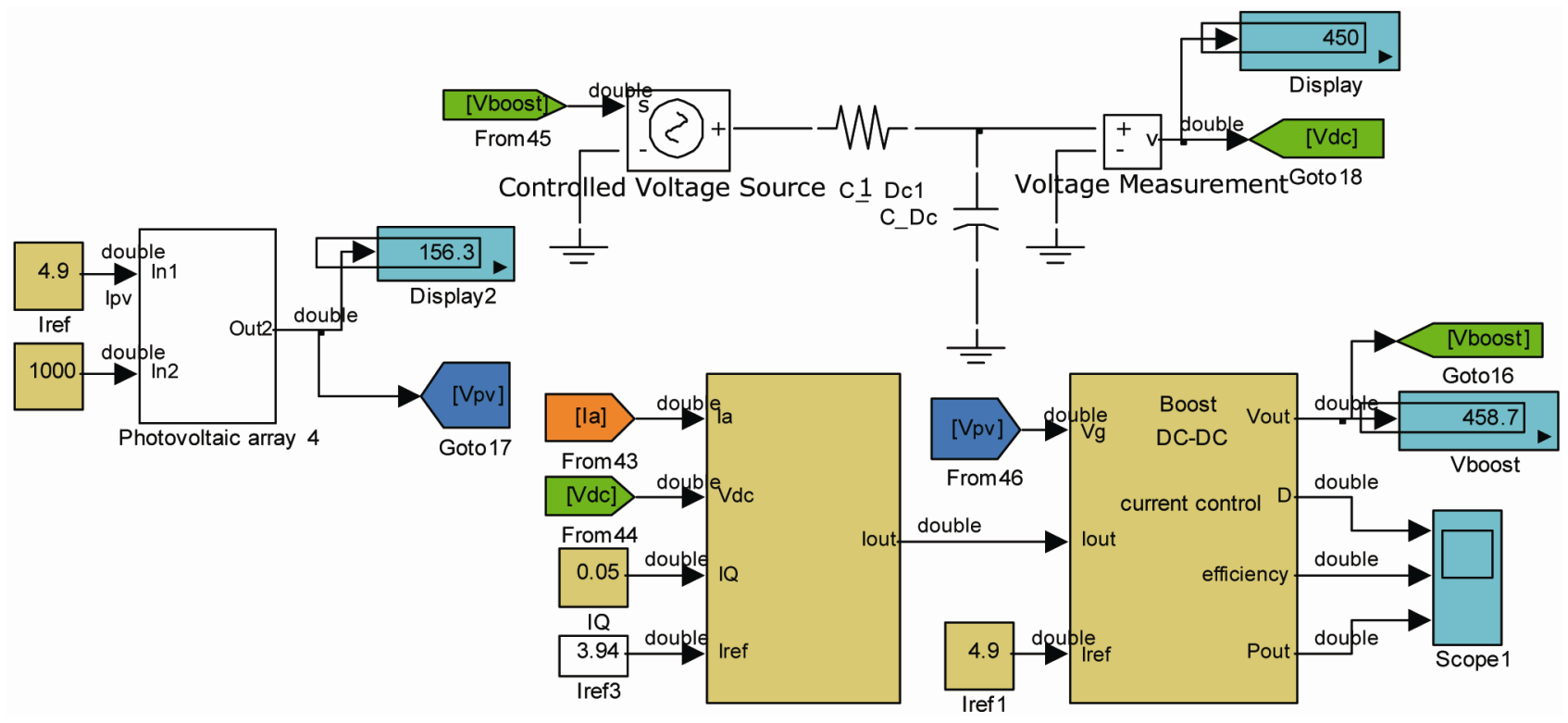

Figure 9. Model of PV using simulink.

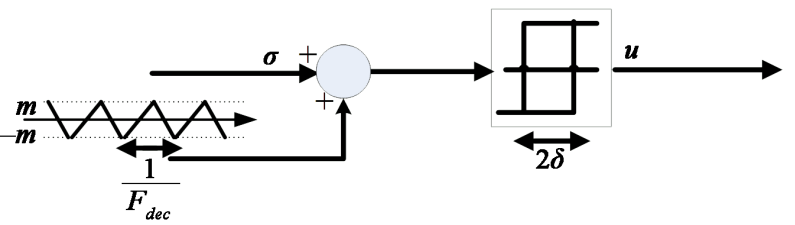

Figure 10. Principle of limiting the switching frequency.

LAB Simulink program. The system parameters are shown in following table. In the simulation, a diode rectifier with a DC-link capacitor and a smoothing inductor was used as a harmonic producing nonlinear load.

The proposed scheme is shown in Figure 11. Now, the high-order harmonics are filtered from the source voltage and source current. The sufficient condition for existence of sliding regimes is the following:

$$
\left\{\begin{array}{l}
\left|u_{d}\right|<\frac{2}{3} \\
\left|u_{q}\right|<\frac{2}{3}
\end{array}\right.
$$

This condition depends on the values chosen for the DC bus voltage $V_{C}$ and the coupling inductance of the inverter network $(L f)$.

The conditions of Equation (26) give:

$$
\left\{\begin{array}{l}
V_{c}>\frac{3}{2}\left(V m+\frac{2 \times L f \times \omega \times I d}{\sqrt{3}}\right) \\
V_{c}>\frac{3}{2}\left(L f \times\left(\frac{2 \times \omega \times I d}{\sqrt{3}} \frac{\cos (\tau)}{\tau}\right)\right)
\end{array}\right.
$$

The equivalent control is then given by:

\begin{tabular}{lc}
\hline \multicolumn{2}{c}{ Matlab Simulink Simulation Parameters } \\
\hline Utility Voltage & $220 \sqrt{2}(50 \mathrm{~Hz})$ \\
Source Inductance & $0.65 \mathrm{e}-3 \mathrm{H}$ \\
Source Resistance & $5^{-3} \Omega$ \\
Rectifier Smoothing Inductor & $1^{-3} \mathrm{H}$ \\
Rectifier Smoothing Resistance & $1.5 \Omega$ \\
Load Inductance & $100^{-3} \mathrm{H}$ \\
Load Resistance & $29 \times 1.5 \Omega$ \\
Load Capacitance & $2000^{-6} \mathrm{~F}$ \\
Current Control Band & $1.8 \mathrm{~A}$ \\
Sampling Time & $10^{-6} \mathrm{~s}$ \\
APF Inductor & $2^{-3} \mathrm{H}$ \\
APF Resistor & $0.005 \Omega$ \\
APF DC-Bus Capacitor & $450 \mathrm{~V}$ \\
HPF Capacitor & $5.01 \mathrm{e}-6 \mathrm{~F}$ \\
HPF Resistor & $20.46 \Omega$ \\
PV & 9 arrays \\
INSOLATION & $1000 \mathrm{w} / \mathrm{m}^{2}$ \\
Short-Circuit Current $\mathrm{I}_{\mathrm{sc}}$ & $5.45 \mathrm{~A}$ \\
Open-Circuit Voltage $\mathrm{V}_{\mathrm{oc}}$ & $22.2 \mathrm{~V}$ \\
Rated Current $\mathrm{I}_{\mathrm{R}}$ at Maximum Power Point (MPP) & $4.95 \mathrm{~A}$ \\
Rated Voltage VR at MPP & $17.2 \mathrm{~V}$ \\
Capacitance of the PV Output & $2^{-3} \mathrm{~F}$ \\
Resistance of PV Output & $72.78 \Omega$ \\
&
\end{tabular}

$$
\begin{aligned}
& u_{d e q}=\frac{L_{f}}{V_{c}}\left(-\frac{\mathrm{d} I_{f d}}{\mathrm{~d} t}+\frac{V_{S d}}{\mathrm{~d} t}-\frac{R_{f}}{L_{f}} I_{f d}+\omega I_{f q}\right) \\
& u_{q e q}=\frac{L_{f}}{V_{c}}\left(-\frac{\mathrm{d} I_{f d}}{\mathrm{~d} t}+\frac{V_{S d}}{\mathrm{~d} t}-\frac{R_{f}}{L_{f}} I_{f d}+\omega I_{f q}\right)
\end{aligned}
$$




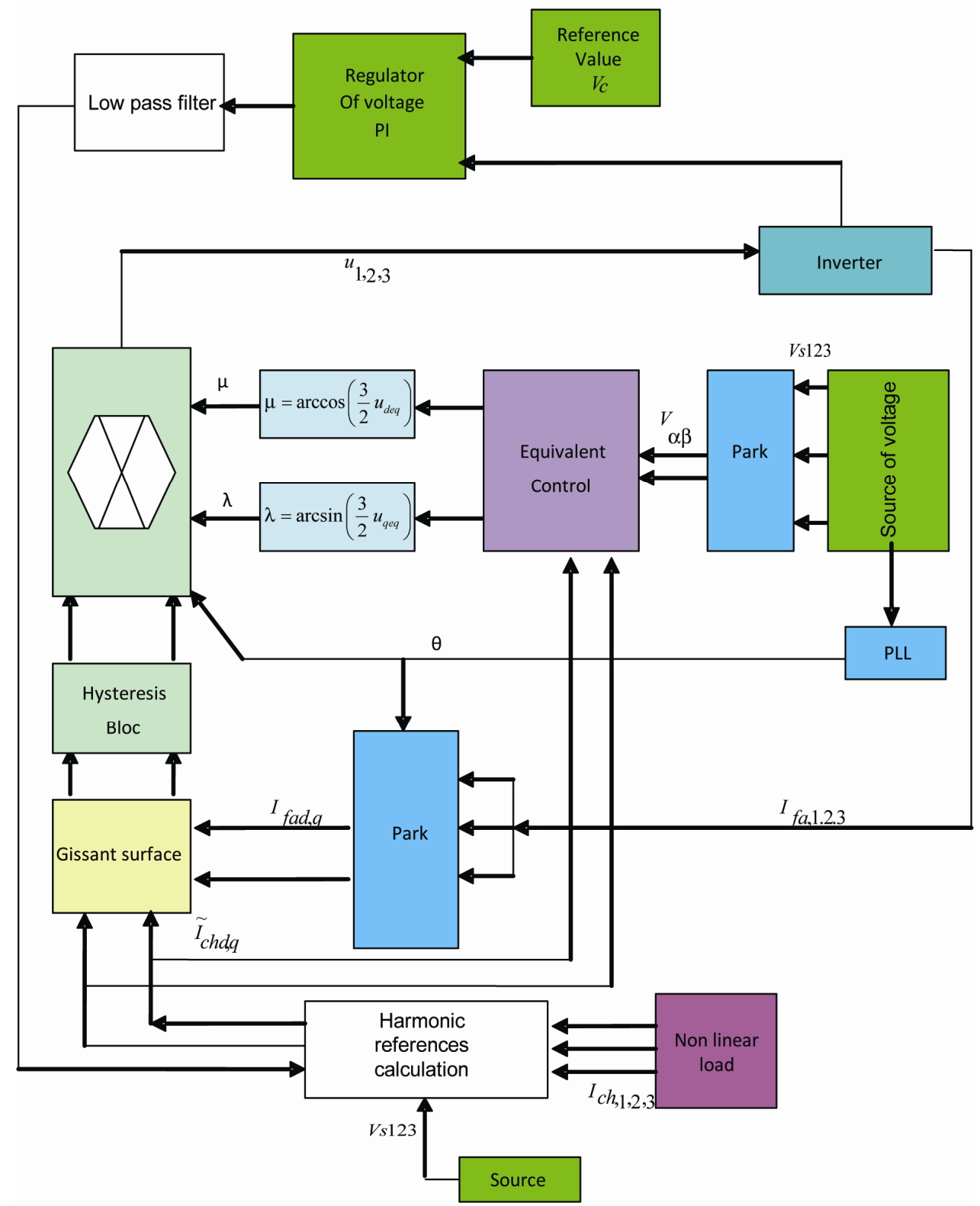

Figure 11. Complete diagram of the control structure

In order to ensure the sliding mode, the value of $V_{c}$ must take the maximum of these two values.

We put:

$$
\lambda=\arcsin \left(\frac{3}{2} u_{q e q}\right) \text { and } \mu=\arccos \left(\frac{3}{2} u_{d e q}\right)
$$

The source currents after compensation are almost sinusoidal and in phase with the voltage at the connection point. The spectra of source current before and after compensation are presented in Figures 12 and 14. The total harmonic distortion (THD) current source (calculated on the first 30 harmonic orders) increased from $30.09 \%$ to $1.95 \%$ and the load current harmonic components are greatly reduced. Note also that the DC bus voltage is regulated to $700 \mathrm{~V}$.

All results are shown in Figure 13 (The source, the current of load before and after the compensation, in addition to the current of the filter and the DC voltage of the capacitor).

Figure 14 shows the spectrum of the source current is in $\mathrm{dB}$. This spectrum allows us to see that the switching frequency varies between 5 and $10 \mathrm{kHz}$. The average switching frequency is equal to $9.07 \mathrm{kHz}$. Low frequency peaks are located between $-40 \mathrm{~dB}$ and $-60 \mathrm{~dB}$. Above 4 $\mathrm{kHz}$, the amplitudes of the lines are growing and both the frequency $7.5 \mathrm{kHz}$ is reached, they are decreasing. Beyond $16 \mathrm{kHz}$, the spectrum is more regular and rays have relative amplitudes below $-50 \mathrm{~dB}$. This spectrum is shown 


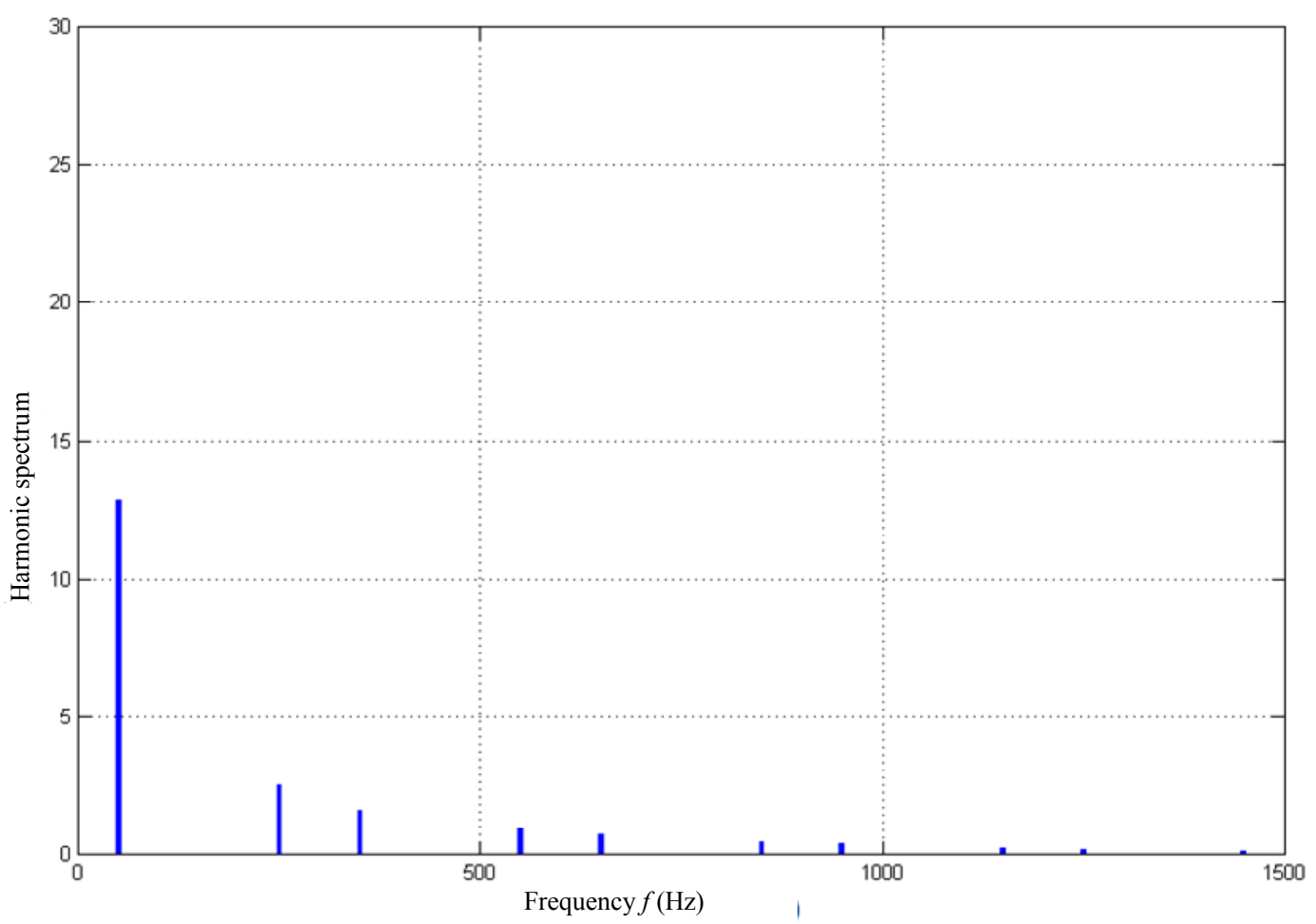

Figure 12. Harmonic spectrum of source current before the compensation.
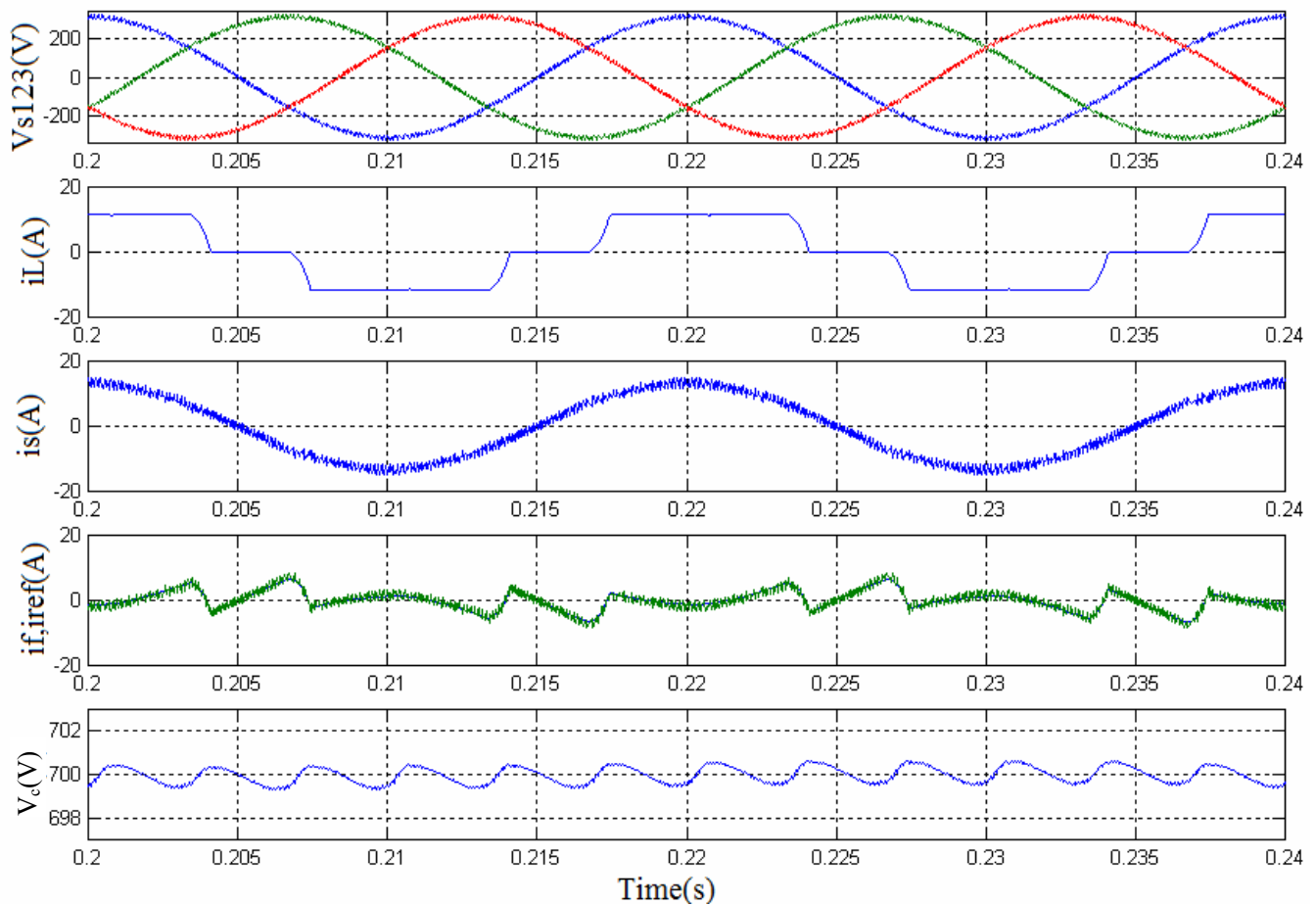

Figure 13. Compensation of harmonic currents with the sliding mode control.

in Figure 15.

\section{Conclusions}

In order to improving of the current reference a signal type sign was added to the equivalent control, the THD is improved (reduced), but the average switching frequency is slightly increased. To reduce the switching frequency without changing system settings, a technique based on 


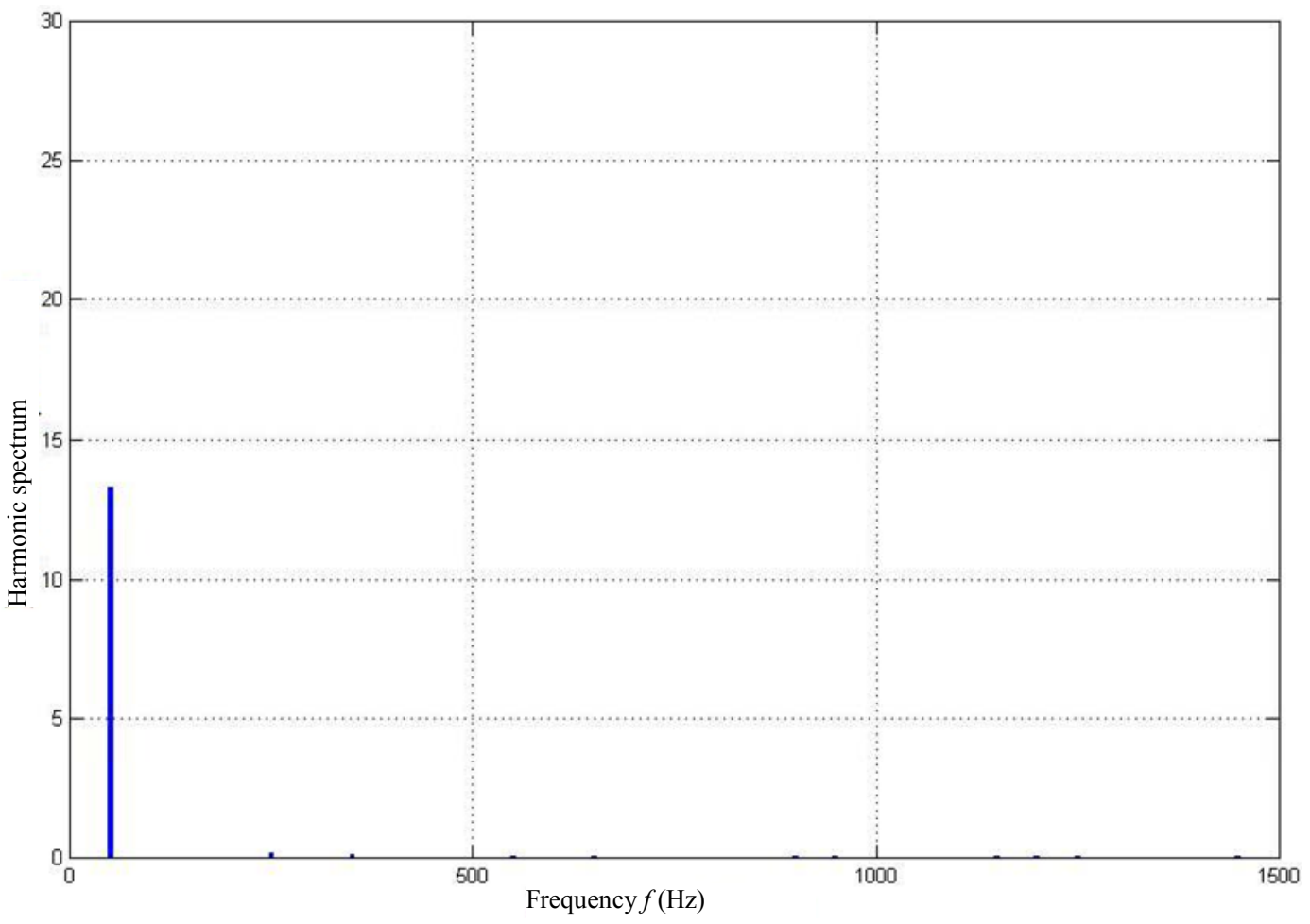

Figure 14. Harmonic spectrum of source current after the compensation.

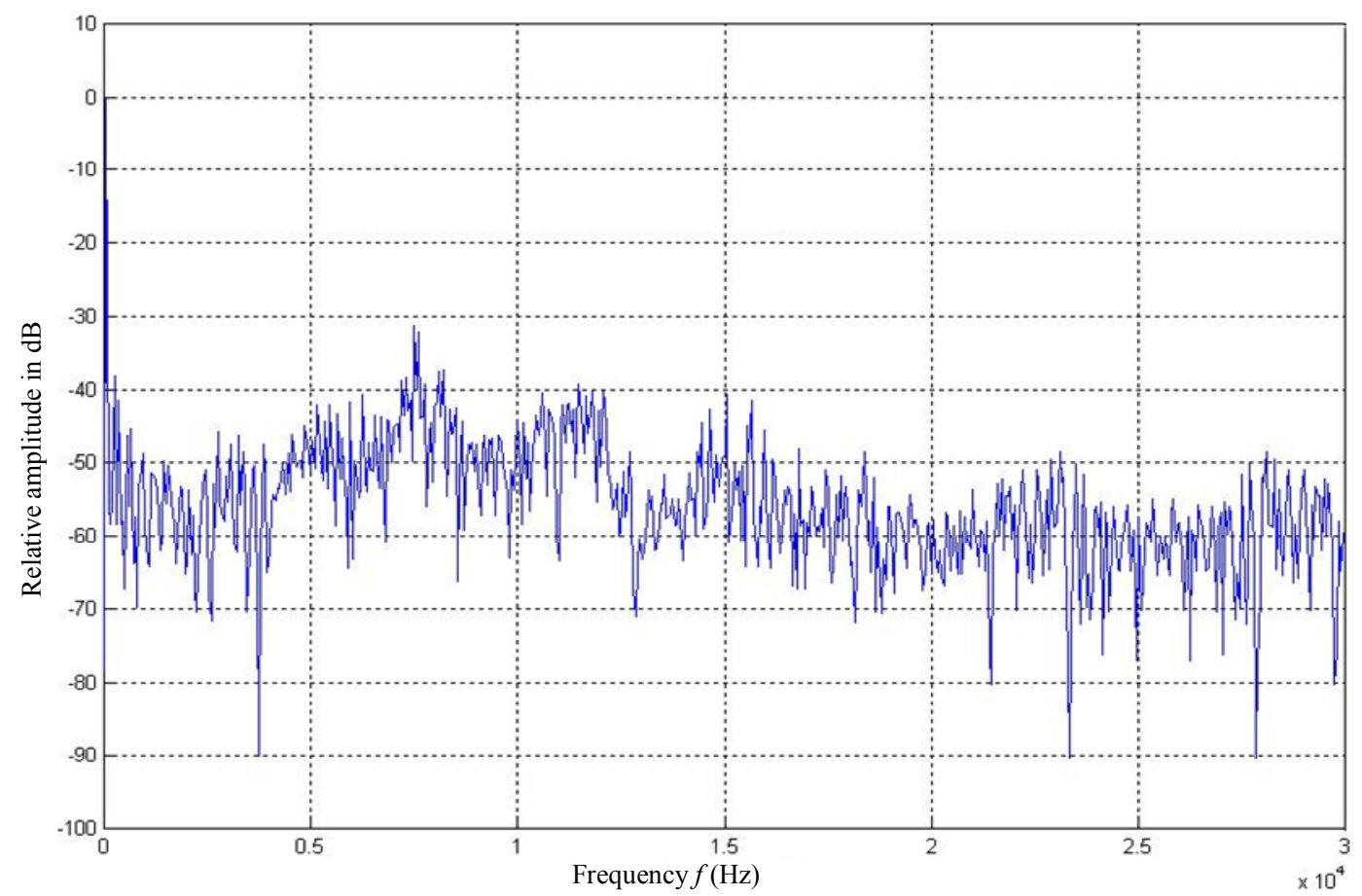

Figure 15. Spectrum of the source current is in $\mathrm{dB}$.

the addition of a triangular signal to the switching surface has been proposed and tested.

The simulation results show the effectiveness of the proposed scheme for wideband harmonics compensation and PV power handling capability. Combined with the fastness and robustness of sliding mode control, HAPF based on sliding mode control has good robustness and stability. The loop for the capacitor voltage regulation is 
introduced into the sliding mode control; good regulation effect in DC bus is obtained for load variation.

\section{REFERENCES}

[1] H. Akagi, "Control Strategy of Active Power Filters Using Multiple Voltage-Source PWM Converters," IEEE Transactions on Industry and Application, Vol. IA-22, No. 3, 1986, pp. 460-465.

[2] A. D. Ould, et al., "A Unified Artificial Neural Network Architecture for Active Power Filters," Industrial Electronics IEEE Transactions on Industrial and Electronics, Vol. 54, No. 1, 2007, pp. 61-76. doi:10.1109/TIE.2006.888758

[3] A. H. S. Rahmani, N. Mendalek and K. Al-Haddad, "A New Control Technique for Three-Phase Shunt Hybrid Power Filter," IEEE Transactions on Industrial Electronics, Vol. 56, No. 8, 2009, pp. 2904-2915. doi:10.1109/TIE.2008.2010829

[4] A. Hamadi, et al., "Sliding Mode Control of Three-Phase Shunt Hybrid Power Filter for Current Harmonics Compensation," International Symposium on Industrial Electronics IEEE, Bari, 4-7 July 2010, pp. 1076-1082.

[5] A. Blorfan, D. Flieller, P. Wira, G. Sturtzer and J. Merckle,

\section{Nomenclature}

HAPF: Hybrid Active Power Filter

APF: Active Power Filter

PV: Photovoltaic

$\sigma(x)$ : Surface of Sliding
"A New Approach for Modeling the Photovoltaic Cell Using Orcad Comparing with the Model Done in Matlab," International Review on Modeling and Simulations, Vol. 3, No. 5, 2010, pp. 948-954.

[6] X.-J. Dai and Q. Chao, "The Research of Photovoltaic Grid-Connected Inverter Based on Adaptive Current Hysteresis Band Control Scheme," Proceedings of the SUPERGEN International Conference on Sustainable Power Generation and Supply, Shanghai, 6-7 April 2009, pp. 1-8.

[7] P. Wang, B.-H. Cheng and Z.-B. Zhang, "Sliding Mode Control for a Shunt Active Power Filter," Proceedings of the $3 r d$ International Conference on Measuring Technology and Mechatronics Automation, Shanghai, 6-7 January 2011, pp. 282-285. doi:10.1109/ICMTMA.2011.641

[8] B. Cheng, et al., "Sliding Mode Control for a Shunt Active Power Filter," Proceedings of the 3rd International Conference on Measuring Technology and Mechatronics Automation, Shanghai, 6-7 January 2011, pp. 282-285.

[9] B. R. Lin and T. Y. Yang, "Three-Level Voltage-Source Inverter for Shunt Active Filter," IEE Transactions on Electric Power Applications, Vol. 151, No. 6, 2004, pp. 744-751. 